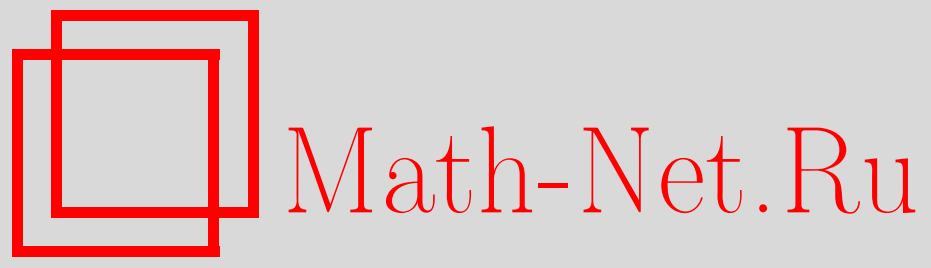

В. П. Маслов, Методы квантовой статистики с точки зрения теории вероятностей. I, Теория вероятн. и ее примен., 2002, том 47, выпуск 4, 686-709

DOI: https://doi.org/10.4213/tvp3775

Использование Общероссийского математического портала MathNet.Ru подразумевает, что вы прочитали и согласны с пользовательским соглашением

http://www . mathnet.ru/rus/agreement

Параметры загрузки:

IP : 34.229 .108 .108

26 апреля 2023 г., 02:54:11

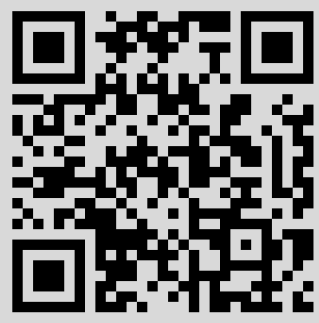




\title{
МЕТОДЫ КВАНТОВОЙ СТАТИСТИКИ С ТОЧКИ ЗРЕНИЯ ТЕОРИИ ВЕРОЯТНОСТЕЙ. I
}

\begin{abstract}
Показано, что стандартным задачам теории вероятностей можно поставить в соответствие самосопряженный оператор в гильбертовом пространстве (в конечномерном случае - симметричную матрицу), ввести понятие энтропии, температуры, а также статистического ансамбля. Ряд общих тождеств для этих величин приводит, в частности, к формулам Купера-Бардина для сверхпроводимости. Строгое доказательство предъявленных асимптотик и их применения будут даны во второй части работы.
\end{abstract}

Ключевые слова и фразы: квантовая термодинамика, финансовая математика, ультравторичное квантование, третичное квантование.

Мы рассмотрим квантованную энтропию в задаче $N$ независимых испытаний при условии, что $N \rightarrow \infty$. При решении вариационной задачи у нас возникает множитель Лагранжа, который аналогичен температуре. Для заданной случайной величины, принимающей дискретные значения, мы получим энтропию как случайную величину.

Заметим, что значения $x_{1}, x_{2}, \ldots, x_{n}$ дискретной случайной величины $X$ обладают следующим свойством. Пусть $p_{1}, p_{2}, \ldots, p_{n}-$ вероятности, отвечающие этим значениям, и пусть $M(X)$ - математическое ожидание $X: M(X)=\sum p_{i} x_{i}$. Так как $p_{i} \geqslant 0$ и $\sum p_{i}=1$, величины $x_{i}$ это экстремумы функционала $M(X)$ по всем $p_{i} \geqslant 0$.

Пусть имеется $N$ независимых испытаний, имеющих следующие исходы (выигрыши):

$$
Y_{N,\left\{K_{i}\right\}}=\sum_{i=1}^{n} x_{i} K_{i}, \quad \sum_{i=1}^{n} K_{i}=N
$$

где $K_{i}$ - положительные целые числа; т.е. порождается случайная величина $Y$, принимающая значения (1). Рассмотрим ситуацию, когда соответствуюшая значению $x_{j}$ вероятность есть $p_{j}=e^{-\gamma x_{\jmath}} / \sum_{k=1}^{n} e^{-\gamma x_{k}}$,

${ }^{*}$ Московский государственный университет им. М. В. Ломоносова, физический факультет, кафедра квантовой статистики, Ленинские горы, 119992 Москва, Россия; e-mail: victor@maslov.msk.su 
где $\gamma$ - некоторая постоянная. Тогда, для случайной величины $Y$, вероятности $\nu$ (биномиальное распределение) равны

$$
\frac{N !}{\prod_{i=1}^{n} K_{i} !} \prod_{i=1}^{n} p_{i}^{K_{i}} .
$$

Введем энтропию Больцмана как случайную величину, принимающую значения

$$
S_{N,\left\{K_{i}\right\}}=\ln \left\{\frac{N !}{\prod_{i=1}^{n} K_{i} !}\right\} .
$$

Пусть $P_{N,\left\{K_{i}\right\}}$ - вероятности, отвечающие случайной величине $Y$, и пусть $\sum P_{N,\left\{K_{i}\right\}}=1$; суммы здесь и далее берутся по всем $\left\{K_{i}\right\}$ таким, что $\sum K_{i}=N$.

Для оценки экстремумов удобнее рассматривать корни из вероятностей. Обозначим $\sigma_{N,\left\{K_{i}\right\}}=\sqrt{P_{N,\left\{K_{i}\right\}}} \ll$ полуплотности», принимающие, вообще говоря, положительные и отрицательные значения. Математическое ожидание величин $Y$ и $S$ тогда можно записать в виде

$$
\begin{gathered}
M(Y)=\sum_{\{K\}} \sigma_{N,\left\{K_{i}\right\}}^{2} Y_{N,\left\{K_{i}\right\}}, \quad M(S)=\sum_{\{K\}} \sigma_{N,\left\{K_{i}\right\}}^{2} S_{N,\left\{K_{i}\right\}}, \\
\sum_{\{K\}} \sigma_{N,\left\{K_{i}\right\}}^{2}=1 .
\end{gathered}
$$

Мы определим случайную величину $S^{\prime}$ и назовем ее относительной энтропией. Ее значения $S_{i}$ определяются как экстремумы математического ожидания энтропии Больцмана по всем $\sigma_{N,\left\{K_{i}\right\}}$ при условии, что математическое ожидание случайной величины $Y$ равно константе, и условии (4). Вероятности, отвечающие этим значениям, равны математическим ожиданиям $\exp \left(S_{N,\left\{K_{i}\right\}}\right)$ по экстремальным значениям $\sigma_{N,\left\{K_{i}\right\}}^{2}$. Аналогично, полагая $M(S)=$ const, ищем экстремумы $M(Y)$ при условии (4). Случайную величину, принимающую эти значения с соответствующими вероятностями, будем называть внутренней энергией. Применяя метод неопределенных множителей Лагранжа, получим экстремум математического ожидания величины

$$
F(\theta)=Y-\theta S
$$

где неопределенный множитель $\theta$ будем называть температурой. Имеет место соотношение $\theta=1 / \gamma$. Естественно полагать, что так же, как и в статистической физике, температура пропорциональна некоторой дисперсии.

Точки экстремума математического ожидания величины $F(\theta)(5)$ могут быть пронумерованы мультииндексом $l=\left(l_{1}, \ldots, l_{n}\right), l_{\alpha}$ - целые 
числа, для которых выполняется условие $\sum_{\alpha=1}^{n} l_{\alpha}=N$. Эти экстремумы имеют вид

$$
\sigma_{N,\{K\}}^{l}=\prod_{\alpha=1}^{n} \delta_{K_{\alpha} l_{\alpha}}, \quad f^{l}(\theta)=Y_{l}-\theta S_{l}
$$

и называются значениями свободной энергии.

Теорема 1. Выполняется соотношение

$$
\lim _{N \rightarrow \infty}\left(\frac{\min _{l} f^{l}(\theta)}{N}\right)=-\theta \ln \left(\sum_{\alpha=1}^{n} e^{-x_{\alpha} / \theta}\right),
$$

где $\min _{l}$ обозначает минимальное из всех значений (6) при фиксированном $\theta$.

Таким образом, при $N \rightarrow \infty$ минимальная свободная энергия определяется формулой Гиббса для свободной энергии. В квантовой физике существует принцип минимальной энергии, который означает, что система со временем приходит в состояние с минимальной энергией. Наша задача - найти другие «энергетические уровни», на которых система будет оставаться достаточно долго (метастабильные состояния). Другими словами, время измерения намного меньше, чем время, за которое система достигает своего окончательного минимального состояния (состояния равновесия).

Пусть далее $I$ - подмножество набора чисел $\alpha=1,2, \ldots, n$, не равное всему набору. Рассмотрим целое число $C>0$ такое, что существует хотя бы один мультииндекс $K$, удовлетворяющий условиям:

$$
K_{\alpha}=0, \quad \text { если } \quad \alpha \in I ; \quad \sum_{\alpha \notin I}\left|K_{\alpha}-K_{\alpha}^{I}(\theta)\right|<C
$$

где числа $K_{\alpha}^{I}(\theta)$ имеют вид:

$$
\begin{array}{ll}
K_{\alpha}^{I}(\theta)=\frac{e^{-x_{\alpha} / \theta}}{\sum_{\beta \notin I} e^{-x_{\beta} / \theta}} N, & \text { если } \\
K_{\alpha}^{I}(\theta)=0, & \text { если } \quad \alpha \in I,
\end{array}
$$

Будем говорить, что те из значений $f^{l}(\theta)$ вида (6), для которых выполняются условия (8), принадлежат $(C, I)$-окрестности. Справедлива следуюшая теорема.

Теорема 2. Для значений $f^{l}(\theta)$, принадлежащих $(C, I)$-окрестности, справедливо неравенство

$$
f^{l}(\theta) \geqslant-N \theta \ln \left(\sum_{\alpha \notin I} e^{-x_{\alpha} / \theta}\right) .
$$


Правую часть неравенства (10) будем называть предельной точкой.

Легко убедиться, что минимальной предельной точкой является правая часть равенства (7), умноженная на $N$.

Рассмотрим число $\Delta(N) \geqslant 0$ такое, что $\Delta(N)=O\left(N^{\delta}\right)$, где $\delta<1$. Введем класс возможных малых шумов математического ожидания случайной величины $F(\theta)(5)$. Будем говорить, что функция от полуплотностей $\sigma_{N,\{K\}}$

$$
V(\sigma)=\sum_{\{k\}} \sum_{\{l\}} V_{\{k\},\{l\}} \sigma_{N,\{k\}} \sigma_{N,\{l\}},
$$

где $\{k\},\{l\}$ - мультииндексы, удовлетворяющие условию $\sum_{\alpha=1}^{n} k_{\alpha}=$ $\sum_{\alpha=1}^{n} l_{\alpha}=N$, принадлежит классу возможных шумов, если выполнены условия

$$
\begin{aligned}
& V_{\{k\},\{l\}}=0 \quad \text { при } \quad \sum_{\alpha=1}^{n}\left|k_{\alpha}-l_{\alpha}\right| \geqslant \Delta, \\
& V_{\{k\},\{l\}}=V_{\{l\},\{k\}}-\text { действительные числа. }
\end{aligned}
$$

Рассмотрим возмущенное математическое ожидание случайной величины $F(\theta)$ :

$$
\widetilde{F}_{\theta, \sigma}=\sum_{\{K\}} f^{K}(\theta) \sigma_{N,\{K\}}^{2}+\varepsilon V(\sigma),
$$

где $V(\sigma)$ имеет вид (11) и является допустимым шумом, а $\varepsilon$ - действительный параметр.

Пусть $f^{l}(\theta, \varepsilon)$ - экстремальные значения функции (13) при дополнительном условии (4), при $\varepsilon=0$ равные (6).

Имеет место следующая теорема о локализаиии.

Теорема 3. Пусть $f^{l}(\theta, \varepsilon)$ - некоторая $(C, I)$-окрестность. Суиествует число $M=O\left(N^{1-\delta}\right)$ такое, что в пределе $n p u \quad N \rightarrow \infty$ $M$ первых членов ряда теории возмущений по параметру $\varepsilon \partial_{л я} f^{l}(\theta, \varepsilon)$, принадлежащего некоторой $(C, I)$-окрестности, не зависят от $f^{k}(\theta)$ $u V_{k m}$, где $k$ и m такие, что $f^{k}(\theta)$ u $f^{m}(\theta)$ принадлежат другой $\left(C^{\prime}, I^{\prime}\right)$ окрестности.

Схема доказательства следующая. Рассматривается функция от полуплотностей, которая имеет вид

$$
V^{M}\left(\sigma, \sigma^{\prime}\right)=\sum_{\{k\}} \sum_{\{l\}} V_{\{k\},\{l\}}^{M} \sigma_{N,\{k\}} \sigma_{N,\{l\}}^{\prime},
$$

где

$$
V_{\{k\},\{l\}}^{M}=\sum_{\left\{n_{1}\right\}} \cdots \sum_{\left\{n_{M-1}\right\}} V_{\{k\},\left\{n_{1}\right\}} V_{\left\{n_{1}\right\},\left\{n_{2}\right\}} \cdots V_{\left\{n_{M-1}\right\},\{l\}} .
$$

В силу условия (12), для (15) справедливо следующее:

$$
V_{\{k\},\{l\}}^{M}=0 \quad \text { при } \quad \sum_{\alpha=1}^{n}\left|k_{\alpha}-l_{\alpha}\right| \geqslant M \Delta .
$$


Из $(16),(8),(9)$ следует, что если $\sigma_{N,\{k\}}^{l}$ и $\sigma_{N,\{k\}}^{m}$ вида (6) отвечают значениям $f^{l}(\theta)$ и $f^{m}(\theta)$, принадлежащим разным $(C, I)$-окрестностям, то

$$
V^{M}\left(\sigma^{l}, \sigma^{m}\right)=0
$$

Члены ряда теории возмущений по параметру $\varepsilon$ для экстремумов функционала $\widetilde{F}_{\theta, \sigma}(13)$ выражаются через функции (14). А именно, $L$ первых членов ряда теории возмущений выражаются через значения функций $V^{M}\left(\sigma, \sigma^{\prime}\right)$ при $M=1, \ldots, L$. Отсюда с учетом (17) может быть строго получено утверждение теоремы.

Покажем, что теорема о локализации может быть проще и изяшнее доказана с помощью введения в вероятностную задачу гильбертова пространства - аналога пространства состояний для квантовых задач, и линейного оператора в этом пространстве - аналога гамильтониана. Сначала рассмотрим простой пример. Пусть случайная величина $X$ принимает два значения $x_{1}$ и $x_{2}$, а соответствующие им вероятности есть $p_{1}$ и $p_{2}$ :

$$
p_{1}=\frac{e^{-\gamma x_{1}}}{e^{-\gamma x_{1}}+e^{-\gamma x_{2}}}, \quad p_{2}=\frac{e^{-\gamma x_{2}}}{e^{-\gamma x_{1}}+e^{-\gamma x_{2}}} .
$$

Рассмотрим линейное пространство $C^{2}$, вект(рами которого являются столбцы из двух элементов

$$
a=\left(\begin{array}{l}
a_{1} \\
a_{2}
\end{array}\right), \quad\|a\|=\sqrt{a_{1}^{2}+a_{2}^{2}} .
$$

Введем в этом пространстве оператор $\widehat{H}_{1}$, который задается диагональной матрицей

$$
H_{1}=\left(\begin{array}{cc}
x_{1} & 0 \\
0 & x_{2}
\end{array}\right)
$$

Пространство $C^{2}$ является аналогом пространства состояний в квантовой задаче, а $\widehat{H}_{1}$ - аналогом гамильтониана в квантовой задаче. Векторы $e_{1}=(1,0)^{\mathrm{T}}, e_{2}=(0,1)^{\mathrm{T}}$ являются собственными векторами оператора $\widehat{H}_{1}$, собственные значения равны $x_{1}$ и $x_{2}$ соответственно. Можно интерпретировать $e_{n}$ как состояние, в котором случайная величина $X$ принимает значение $x_{n}$. Рассмотрим серию из $N$ независимых испытаний, в каждом из которых возможный исход - это случайная величина $X$. Соответствующая серии из $N$ независимых испытаний случайная величина $Z$ принимает значения $\left(x_{k_{1}}, \ldots, x_{k_{N}}\right)$ - наборы из $N$ чисел, $k_{j}=1,2$; соответствующие вероятности имеют вид

$$
P_{k_{1}, \ldots, k_{N}}=p_{k_{1}} \cdots p_{k_{N}}
$$


Кроме $Z$ будем также рассматривать случайную величину $Y$ - выигрыш (1). Перенесем аналогию пространства состояний и гамильтониана в задачу о $N$ независимых испытаниях. Аналогом пространства состояний будем считать пространство

$$
C^{2 N}=C^{2} \otimes C^{2} \otimes \cdots \otimes C^{2},
$$

векторами которого являются столбцы из $2 N$ элементов. Рассмотрим в $C^{2 N}$ оператор $\widehat{H}_{N}$, который задается формулой

$$
\widehat{H}_{N}=\sum_{s=1}^{N} \widehat{E} \otimes \cdots \otimes \widehat{E} \otimes \widehat{H}_{1} \otimes \widehat{E} \otimes \cdots \otimes E,
$$

где $\widehat{E}$ - единичный оператор в пространстве $C^{2}$, каждое слагаемое представляет собой тензорное произведение $N-1$ операторов $\widehat{E}$ и оператора $\widehat{H}_{1}$, причем в слагаемом с номером $s$ оператор $\widehat{H}_{1}$ в произведении стоит на $s$-м месте. Собственные векторы оператора (21) имеют вид

$$
f_{k_{1}, \ldots, k_{N}}=e_{k_{1}} \otimes e_{k_{2}} \otimes \cdots \otimes e_{k_{N}}
$$

а соответствующие собственные значения есть

$$
Y_{k_{1}, \ldots, k_{N}}=x_{k_{1}}+x_{k_{2}}+\cdots+x_{k_{N}} .
$$

Аналогично (18) рассмотрим выражение

$$
P_{k_{1}, \ldots, k_{N}}=\frac{e^{-\gamma Y_{k_{1}, \ldots, k_{N}}}}{\sum_{k_{1}^{\prime}=1}^{2} \cdots \sum_{k_{N}^{\prime}=1}^{2} e^{-\gamma Y_{k_{1}^{\prime}, \ldots, k_{N}^{\prime}}}} .
$$

В силу (23) очевидно, что выражение (24) совпадает с (20). Пусть $x_{1} \neq x_{2}$, тогда формула (23) может быть переписана в виде

$$
Y_{k_{1}, \ldots, k_{N}}=x_{1} N_{1}+x_{2} N_{2}
$$

где $N_{1}, N_{2}$ - целые числа, зависящие от набора $\left(k_{1}, \ldots, k_{N}\right)$ следующим образом:

$$
N_{1}=\sum_{s=1}^{N} \delta_{1 k_{s}}, \quad N_{2}=\sum_{s=1}^{N} \delta_{2 k_{s}},
$$

здесь $\delta_{k l}$ - символ Кронекера. Заметим, что $N_{1}+N_{2}=N$. Выражение (25) совпадает с выигрышем $Y_{N,\left\{N_{1}, N_{2}\right\}}(1)$. Поскольку для разных наборов $\left(k_{1}, \ldots, k_{N}\right)$ выражения (26) могут совпадать, собственные значения оператора $\hat{H}_{N}$ вырождены, кратность вырождения собственного значения (25) равна $N ! /\left(N_{1} ! N_{2} !\right)$. Очевидна связь оператора (21) в пространстве $C^{2 N}$ с вероятностной задачей о $N$ независимых испытаниях и выигрыше. Возможные значения выигрыша равны собственным 
значениям оператора $\widehat{H}_{N}$, а вероятности выигрыша, которые выражаются формулой (2), равны кратности собственных значений, умноженной на (24). Таким образом, вероятностной задаче сопоставлены гильбертово пространство и оператор в этом пространстве. Введем в пространстве $C^{2 N}$ оператор $\widehat{S}_{N}$, собственные векторы которого суть $(22)$, а соответствующие собственные значения

$$
S_{k_{1}, \ldots, k_{N}}=\ln \left(\frac{N !}{N_{1} ! N_{2} !}\right),
$$

где $N_{1}, N_{2}$ заданы формулой (26). Очевидно, что собственные значения операторов $\widehat{H}_{N}, \widehat{S}_{N}$ и $\widehat{F}_{N, \theta}=\widehat{H}_{N}-\theta \widehat{S}_{N}$ совпадают с экстремальными значениями математических ожиданий $M(Y), M(S)$ (3) и математического ожидания величины $F(\theta)(5)$ соответственно. Предположим, что на серию испытаний воздействует шум, который приводит к тому, что испытания не являются независимыми. Сопоставим шуму оператор $\varepsilon \widehat{V}$ в пространстве $C^{2 N}$. Будем считать, что вероятностная задача о выигрыше в серии испытаний, находяшейся под воздействием шума, описывается оператором $\widehat{F}_{N, \theta}^{\varepsilon}=\widehat{F}_{N, \theta}+\varepsilon \widehat{V}$, т.е. возможные значения выигрыша равны собственным значениям оператора $\widehat{F}_{N, \theta}^{\varepsilon}$, а вероятности выигрышей выражаются через собственные значения по формуле, аналогичной (24). Собственные векторы оператора $\widehat{F}_{N, \theta}^{\varepsilon}$ не совпадают с векторами (22). Собственные значения оператора $\widehat{F}_{N, \theta}^{\varepsilon}$ совпадают с экстремальными значениями функционала (13) в случае, если

$$
V_{\{k\},\{l\}}=\left(f_{k_{1}, \ldots, k_{N}}, \widehat{V} f_{l_{1}, \ldots, l_{N}}\right) \text {. }
$$

Отсюда следует, что для доказательства теоремы о локализации достаточно доказать аналог этой теоремы для $\widehat{F}_{N, \theta}^{\varepsilon}$, а для этого можно применить стандартную схему теории возмушений. Рассмотренная схема сопоставления вероятностной задаче гильбертова пространства и оператора без затруднений переносится на общий случай. Например, в случае, когда в серии из $N$ испытаний случайная величина $X$ принимает $n$ значений $x_{1}, \ldots, x_{n}$, соответствующее гильбертово пространство для одного испытания есть $C^{n}$, а оператор $\widehat{H}_{1}$ задается матрицей размера $n \times n$, у которой на главной диагонали стоят числа $x_{1}, \ldots, x_{n}$, а остальные элементы равны 0 , соответствующие собственные векторы будем обозначать $e_{1}, \ldots, e_{n}$. Гильбертово пространство, отвечающее серии из $N$ испытаний, в этом случае есть $C^{n N}$, а гамильтониан $\widehat{H}_{N}$ выражается через $\widehat{H}_{1}$ по формуле $(21)$. Собственные значения оператора $\widehat{H}_{N}$ совпадают со значениями выигрыша (1), а вероятность соответствуюшего исхода серии из $N$ испытаний выражается через собственные значения по формуле, аналогичной (24). Кратность собственного значения $Y=x_{1} N_{1}+\cdots+x_{n} N_{n}$ в случае, когда числа $x_{1}, \ldots, x_{n}$ несоизмеримы, 
равна $N ! /\left(N_{1} ! \cdots N_{n} !\right)$. Вероятность выигрыша, равная произведению кратности и вероятности, полученной по формуле, аналогичной $(24)$, совпадает с (2).

Рассмотрим теперь обобщения вероятностной задачи о выигрыше в серии из $N$ независимых испытаний. Введение гильбертова пространства и оператора позволяет решить эти новые вероятностные задачи очень просто. Рассмотрим выигрыш в серии из $N$ испытаний, причем объединим в одну серию все серии, у которых совпадают выигрыши, а различие состоит в порядке выпадения исходов $x_{1}, \ldots, x_{n}$. В некотором смысле можно считать, что испытания тождественны и от их перестановки ничего не меняется. В такой вероятностной задаче сериям можно взаимнооднозначно сопоставить наборы из $n$ неотрицательных целых чисел $\{K\}=\left(K_{1}, \ldots, K_{n}\right)$ таких, что $K_{1}+\cdots+K_{n}=N$. Выигрыш, соответствующий набору $\{K\}$, выражается формулой (1), а вероятность этого выигрыша равна

$$
P_{\{K\}}=\frac{p_{1}^{K_{1}} \cdots p_{n}^{K_{n}}}{\sum_{\left\{K^{\prime}\right\}} p_{1}^{K_{1}^{\prime}} \cdots p_{n}^{K_{n}^{\prime}}} .
$$

Сопоставим этой новой задаче пространство $C_{\mathrm{S}}^{n N}$, которое является подпространством пространства $C^{n N}$ и состоит из его элементов, имеющих вид

$$
f=\sum_{m_{1}=1}^{n} \cdots \sum_{m_{N}=1}^{n} f\left(m_{1}, \ldots, m_{N}\right) \cdot e_{m_{1}} \otimes \cdots \otimes e_{m_{N}}
$$

где $f\left(m_{1}, \ldots, m_{N}\right)$ - числовые коэффициенты, симметричные относительно перестановок $m_{s}$ и $m_{r}, s, r=1, \ldots, n, e_{m}$ - собственные векторы оператора $\widehat{H}_{1}$. Пространство $C_{\mathrm{S}}^{n N}$ будем называть симметричным подпространством пространства $C^{n N}$. Соответствующий оператор есть ограничение введенного выше оператора $\widehat{H}_{N}$ на подпространство $C_{\mathrm{S}}^{n N}$. Легко убедиться, что собственные значения этого оператора совпадают со значениями выигрыша, их кратность равна 1, а вероятность выигрыша (28) может быть выражена через собственные значения по формуле, аналогичной (24). Отметим, что такая постановка задачи о выигрыше в серии испытаний имеет практическое значение. Например, таким способом можно рассматривать задачу о распределении купюр одного достоинства по пачкам.

Рассмотрим еще одну задачу о выигрыше в серии из $N$ испытаний. Так же, как в предыдущей задаче, будем считать, что серии с одинаковым выигрышем, отличающиеся только перестановкой исходов $x_{m_{1}}, x_{m_{2}}, \ldots, x_{m_{N}}, m_{j}=1, \ldots, n$, объединяются в одну серию, а кроме того, наложим запрет на серии, в которых хотя бы один из исходов $x_{1}, \ldots, x_{n}$ всгречается больше одного раза. В такой задаче сериям взаимнооднозначно сопоставляются наборы из $n$ неотрицательных целых 
чисел $\{K\}=\left(K_{1}, \ldots, K_{n}\right)$ таких, что $0 \leqslant K_{j} \leqslant 1, \sum_{i=1}^{n} K_{i}=N$. Выигрыш, отвечающий набору $\{K\}$, выражается формулой (1), а соответствующие вероятности выражаются формулой (28), в которой надо учесть условие $0 \leqslant K_{j}^{\prime} \leqslant 1$. Пространством, отвечающим такой постановке вероятностной задачи о выигрыше в серии из $N$ испытаний, является $C_{\mathrm{A}}^{n N}$ - подпространство пространства $C^{n N}$, состояшее из его элементов, представимых в виде $(29)$, где коэффициенты $f\left(m_{1}, \ldots, m_{N}\right)$ антисимметричны относительно перестановок $m_{s}$ и $m_{r}, s, r=1, \ldots, N$. Будем называть $C_{\mathrm{A}}^{n N}$ антисимметричным подпространством пространства $C^{n N}$. Оператором в данном случае является ограничение оператора $\widehat{H}_{N}$ на подпространство $C_{\mathrm{A}}^{n N}$. Так же, как и в предыдущих случаях, собственные значения оператора совпадают со значениями выигрыша, а вероятности выражаются через собственные значения формулой, аналогичной (24). Практическим применением такой постановки задачи о выигрыше в серии испытаний является рассмотрение задачи о распределении брокеров по биржам так, чтобы на бирже находилось не более одного брокера, или задачи о расстановке шашек на доске так, что на одно поле помещается не более одной шашки.

Рассмотренные задачи о серии испытаний принципиально отличаются одна от другой: в рассмотренных задачах существует различие между собственными функциями оператора $\widehat{H}_{N}$. Собственные функции вида (22) обладают следующим свойством: если представить их в виде столбца, состоящего из $n N$ элементов, то квадрат каждого элемента равен сам себе, поскольку в такой записи векторы (22) представляют собой столбцы из 0 и 1 . Поэтому в обычной задаче о серии независимых испытаний полуплотность совпадает с плотностью вероятности. В случае, когда собственные функции принадлежат симметричному $C_{\mathrm{S}}^{n N}$ или антисимметричному $C_{\mathrm{A}}^{n N}$ подпространству, это свойство не выполняется и полуплотность не равна плотности вероятности. Таким образом, введенное пространство и оператор оказались существенными.

Анализируя результат теоремы 3 , мы прежде всего придем к некоторой естественной классификации случайных величин.

Пусть случайная величина принимает значения $\lambda_{1}$ и $\lambda_{2}$, а $p_{1}$ и $p_{2}$ отвечающие им вероятности, $p_{1}+p_{2}=1$. Сопоставим $\lambda_{1}$ и $\lambda_{2}$ соответственно два случайных $k$-мерных вектора, вероятности компонент которых равны $p_{1}^{i}, i=1, \ldots, k$, и $p_{2}^{i}, i=1, \ldots, k, \sum_{i=1}^{k} p_{1}^{i}=p_{1}, \sum_{i=1}^{k} p_{2}^{i}=p_{2}$.

Будем называть $\lambda_{1}$ и $\lambda_{2}$ состояниями. Рассмотрим симметричную действительную $(k \times k)$-матрицу $A$ и будем говорить, что математическое ожидание этой матрицы в состоянии $\lambda_{1}$ (и отвечающего ему вектора) равно $M_{\lambda_{1}}(A)=(\bar{\alpha}, A \bar{\alpha})$, где $\bar{\alpha}$ - вектор с компонентами $\bar{\alpha}=\left(\sqrt{p_{1}^{1}}, \ldots, \sqrt{p_{1}^{k}}\right)$. Соответственно, математические ожидания матрицы $A$ в состоянии $\lambda_{2}$ суть $M_{\lambda_{1}}(A)=(\bar{\beta}, A \bar{\beta})$, где $\bar{\beta}=\left(\sqrt{p_{2}^{1}}, \ldots, \sqrt{p_{2}^{k}}\right)$. Вероятность перехода из состояния $\lambda_{1}$ в состояние $\lambda_{2}$ определим как 
$M_{\lambda_{1} \lambda_{2}}(A)=(\bar{\alpha}, A \bar{\beta})$. Пусть $p_{1}^{i}=0$ при $k>i \geqslant s$ и $p_{2}^{i}=0$ при $i<s$. Если матрица имеет вид $\left(\begin{array}{cc}A_{1} & 0 \\ 0 & A_{2}\end{array}\right)$, где $A_{1}$ и $A_{2}$ - матрицы соответственно в $s$-мерном и $(k-s-1)$-мерном пространствах, то $(\bar{\alpha}, A \bar{\beta})=0$ и, тем самым, задача может быть разделена на два ортогональных подпространства.

Будем говорить, что состояния $\lambda_{1}$ и $\lambda_{2}$ независимы, если $(\alpha, E \beta)=0$, где $E$ - единичная матрица («белый шум»).

Рассмотрим в качестве примера пространство $L_{2}(\Omega)$. Пусть $\widehat{A}$ дифференциальный оператор, а носители $\Omega_{1}$ и $\Omega_{2}, \Omega_{1}+\Omega_{2}=\Omega$, вероятностных распределений не пересекаются. Тогда $\int \sqrt{p_{1}(x)} A \sqrt{p_{2}(x)} d x=$ 0 , а это и означает, что пространство разбивается на $L_{2}\left(\Omega_{1}\right)$ и $L_{2}\left(\Omega_{2}\right)$.

3 а м е ч а и е. Корень квадратный из вероятности $\sqrt{p}$ может быть как положительным, так и отрицательным. На математическом ожидании состояния $\lambda_{1}$ это не сказывается, но на математическом ожидании перехода из $\lambda_{1}$ в $\lambda_{2}$ это существенно сказывается и является некоторой дополнительной характеристикой состояния. В дальнейшем мы будем иметь дело только с бесконечномерными векторами, зависящими от непрерывной переменной, и требование достаточной гладкости корня из распределения снимает эту проблему.

Теперь рассмотрим случайный вектор $x \in \mathbf{R}^{3}$, зависящий от дискретной случайной величины $n$, принимающей значения $0,1, \ldots$. Пусть $p_{n}(x)$ - отвечающая ему плотность вероятности

$$
\int d x p_{n}(x)=p_{n}>0, \quad \sum_{n=0}^{\infty} \int d x p_{n}(x)=1 .
$$

Будем считать, что выполнены условия:

1) существует $\varphi_{n}(x)$ - гладкая функция параметров $x$, называемая полуплотностью, такая, что $p_{n}(x)=\left|\varphi_{n}(x)\right|^{2}$;

2) $\int d x \varphi_{i}(x) \varphi_{n}(x)=0$, если $i \neq n$, т.е. при разных значениях дискретной случайной величины отвечающие им состояния независимы;

3) система $\left\{\varphi_{n}(x)\right\}$ полна в гильбертовом пространстве $L_{2}\left(\mathbf{R}^{3}\right)$.

Рассмотрим в пространстве $L_{2}\left(\mathbf{R}^{3}\right)$ оператор $\widehat{H}$ вида

$$
(\widehat{H} \varphi)(x)=-\frac{1}{\gamma} \sum_{i=0}^{\infty}\left(\frac{\ln p_{i}}{p_{i}}\right) \varphi_{i}(x) \int d y \varphi_{i}(y) \varphi(y)
$$

Очевидно, что $\varphi_{n}(x)$ есть собственная функция оператора $\widehat{H}$, соответствующее собственное значение равно $\lambda_{n}=-\frac{1}{\gamma} \ln p_{n}$. Вероятность того, что дискретная случайная величина $n$ принимает значение $i$, равна $p_{i}=\int d x\left|\varphi_{n}(x)\right|^{2}=e^{-\gamma \lambda_{n}}$, т.е. выражается как через собственные значения, так и через собственные функции оператора (30). 
Введем понятие ансамбля для вероятностных задач. Ансамблем, отвечающим вероятностной задаче, определяемой оператором (30), будем называть серию из $N$ независимых испытаний, в каждом из которых исход - рассмотренный выше случайный вектор $x \in \mathbf{R}^{3}$, зависящий от случайной величины $n$. Такой серии $N$ испытаний отвечает случайный вектор $\left(x_{1}, \ldots, x_{N}\right)=X \in \mathbf{R}^{3 N}$, зависящий от дискретной случайной величины $\left(n_{1}, \ldots, n_{N}\right)$. В силу независимости испытаний, соответствующая плотность вероятности есть

$$
\mathscr{P}_{n_{1}, \ldots, n_{N}}\left(x_{1}, \ldots, x_{N}\right)=p_{n_{1}}\left(x_{1}\right) \cdots p_{n_{N}}\left(x_{N}\right)
$$

Для функций (31) выполняются условия, аналогичные 1)-3). А именно, гладкие функции

$$
\Phi_{n_{1}, \ldots, n_{N}}\left(x_{1}, \ldots, x_{N}\right)=\varphi_{n_{1}}\left(x_{1}\right) \cdots \varphi_{n_{N}}\left(x_{N}\right)
$$

образуют полную систему в $L_{2}\left(\mathbf{R}^{3 N}\right)$ и таковы, что

$$
\begin{gathered}
\mathscr{P}_{n_{1}, \ldots, n_{N}}\left(x_{1}, \ldots, x_{N}\right)=\left|\Phi_{n_{1}, \ldots, n_{N}}\left(x_{1}, \ldots, x_{N}\right)\right|^{2} \\
\int \cdots \int d x_{1} \cdots d x_{N} \Phi_{n_{1}, \ldots, n_{N}}\left(x_{1}, \ldots, x_{N}\right) \Phi_{n_{1}^{\prime}, \ldots, n_{N}^{\prime}}\left(x_{1}, \ldots, x_{N}\right)=0
\end{gathered}
$$

если $\left(n_{1}, \ldots, n_{N}\right) \neq\left(n_{1}^{\prime}, \ldots, n_{N}^{\prime}\right)$. Вероятности, отвечающие дискретной случайной величине $\left(n_{1}, \ldots, n_{N}\right)$, суть

$$
\mathscr{P}_{n_{1}, \ldots, n_{N}}=\int \cdots \int d x_{1} \cdots d x_{N} \mathscr{P}_{n_{1}, \ldots, n_{N}}\left(x_{1}, \ldots, x_{N}\right)=p_{n_{1}} \cdots p_{n_{N}}
$$

Рассмотрим в пространстве $L_{2}\left(\mathbf{R}^{3 N}\right)$ оператор $\widehat{H}_{n}$, который есть сумма операторов (30):

$$
\begin{aligned}
\left(\widehat{H}_{n} \Phi\right)\left(x_{1}, \ldots, x_{N}\right)= & -\frac{1}{\gamma} \sum_{n=0}^{\infty} \frac{\ln p_{n}}{p_{n}} \sum_{s=1}^{N} \varphi_{n}\left(x_{s}\right) \\
& \times \int d y \varphi_{n}(y) \Phi\left(x_{1}, \ldots, x_{s-1}, y, x_{s+1}, \ldots, x_{N}\right) .
\end{aligned}
$$

Функции (32) являются собственными функциями оператора $\widehat{H}_{N}$, соответствующие собственные значения суть

$$
\Lambda_{n_{1}, \ldots, n_{N}}=-\frac{1}{\gamma} \ln p_{n_{1}}-\cdots-\frac{1}{\gamma} \ln p_{n_{N}}=-\frac{1}{\gamma} \ln \mathscr{P}_{n_{1}, \ldots, n_{N}} .
$$

Таким образом, свойства оператора (34), введенного для серии независимых испытаний, аналогичны свойствам оператора (30), введенного для исходной случайной величины. 
Эти операторы позволяют определить распределения вероятностей в соответствующих случаях, поэтому далее мы будем формулировать вероятностную задачу с помощью задания оператора. Рассмотрим теперь простое обобщение ансамбля. Будем считать, что испытания тождественны, т.е. от перестановки испытаний местами случайная величина не меняется, тогда плотность вероятности $\mathscr{P}_{n_{1}, \ldots, n_{N}}\left(x_{1}, \ldots, x_{N}\right)$ является симметричной функцией относительно перестановки любой пары $x_{s}$ и $x_{r}$. Это означает, что соответствующая полуплотность $\Phi_{n_{1}, \ldots, n_{N}}\left(x_{1}, \ldots, x_{N}\right)$ является симметричной или антисимметричной функцией относительно перестановок пар $x_{s}$ и $x_{r}$. Для функций (32) это не выполняется, кроме того, испытания, вообще говоря, уже нельзя считать независимыми. Поэтому сформулируем вероятностную задачу следующим образом. Будем считать, что полуплотности $\Phi_{\alpha}\left(x_{1}, \ldots, x_{N}\right)$ являются в случае симметричного ансамбля симметричными собственными функциями оператора $\widehat{H}_{N}(34)$, а в случае антисимметричного ансамбля - антисимметричными, причем такими, что

$$
\begin{aligned}
& \int d x_{1} \cdots d x_{N} \Phi_{\alpha}\left(x_{1}, \ldots, x_{N}\right) \Phi_{\beta}\left(x_{1}, \ldots, x_{N}\right) \\
& =\left\{\begin{array}{lll}
0 & \text { при } & \alpha \neq \beta, \\
\frac{e^{-\gamma \Lambda_{\alpha}}}{\sum_{\sigma} e^{-\gamma \Lambda_{\sigma}}} & \text { при } & \alpha=\beta,
\end{array}\right.
\end{aligned}
$$

где $\Lambda_{\alpha}$ - соответствуюшее $\Phi_{\alpha}$ собственное значение оператора (34). Тогда $\mathscr{P}_{\alpha}\left(x_{1}, \ldots, x_{N}\right)=\left|\Phi_{\alpha}\left(x_{1}, \ldots, x_{N}\right)\right|^{2}$ есть плотность вероятности для случайного вектора $\left(x_{1}, \ldots, x_{N}\right)=X \in \mathbf{R}^{3 N}$, зависящего от дискретной случайной величины $\alpha$ (эта случайная величина - «номер» собственной функции). В симметричном случае в качестве $\alpha$ удобно выбрать бесконечные наборы целых чисел, из которых только конечное число отлично от нуля: $\alpha=\left(K_{0}, K_{1}, K_{2}, \ldots\right)$, где $K_{i} \geqslant 0$ - целые числа такие, что $\sum_{i=0}^{\infty} K_{i}=N$. Пронумерованные таким образом симметричные собственные функции и собственные значения оператора (34) имеют вид

$$
\begin{aligned}
& \Lambda_{\alpha}=-\frac{1}{\gamma} \ln \left(\prod_{i=0}^{\infty} p_{i}^{K_{i}}\right) \\
& \Phi_{\alpha}\left(x_{1}, \ldots, x_{N}\right)=C_{\alpha} \cdot \underset{\left(x_{1}, \ldots, x_{N}\right)}{\operatorname{Symm}}\left(\varphi_{0}\left(x_{1}\right) \cdots \varphi_{0}\left(x_{K_{0}}\right) \cdots\right. \\
&\left.\quad \times \varphi_{1}\left(x_{K_{0}+1}\right) \cdots \varphi_{1}\left(x_{K_{0}+K_{1}}\right) \cdots\right),
\end{aligned}
$$

где $\operatorname{Symm}_{\left(x_{1}, \ldots, x_{N}\right)}$ - оператор симметризации по переменным $x_{1}, \ldots, x_{N}$, а $C_{\alpha}$ - коэффициент, который находится из условия (36). В антисимметричном случае способ нумерации аналогичный, а числа $K_{i}$ удовлетворяют дополнительному условию: $K_{i} \leqslant 1$. Собственные значения и собственные функции в антисимметричном случае выражаются форму- 
лами (37) и (38), только оператор симметризации надо заменить на оператор антисимметризации $\operatorname{Asymm}_{\left(x_{1}, \ldots, x_{N}\right)}$.

Пусть $z \in \cdot \mathbf{R}$ - одномерная непрерывная случайная величина, $\rho(z)$ - соответствующая плотность вероятности. Вероятность того, что $z$ находится в интервале $(a, b)$, равна

$$
\mathbf{P}\{a<z<b\}=\int_{a}^{b} d z \rho(z) .
$$

В случае, когда непрерывная случайная величина зависит от дискретной случайной величины, как введенный выше случайный вектор $x \in \mathbf{R}^{3}$, зависящий от $n=0,1, \ldots$, естественно рассматривать вероятности того, что $x$ находится в некоторой области $\Omega$, а $n$ не превышает $j$ и не меньше, чем $i$. Такая вероятность имеет вид

$$
\mathbf{P}\{x \in \Omega ; i \leqslant n \leqslant j\}=\sum_{n=i}^{j} \int_{\Omega} d x p_{n}(x) .
$$

Если $\Omega=\mathbf{R}^{3}$, то эта формула выражает вероятность того, что дискретная случайная величина имеет значение $n$ такое, что $i \leqslant n \leqslant j$, а при дополнительном условии $i=j$ это выражение равно $p_{i}-$ вероятности того, что $n=j$. Формула (39) естественным образом переносится на случай ансамбля. Представим множество чисел $0,1,2, \ldots$ в виде объединения непересекающихся интервалов $I_{s}, s=1,2, \ldots$. Пусть в интервале $I_{s}$ находится $G_{s}$ чисел, т.е. $j \in I_{s}$, если $G_{1}+\cdots+G_{s-1}-1<j \leqslant$ $G_{1}+G_{2}+\cdots+G_{s}-1$. Введем следующие случайные величины:

$$
Z_{s}=\sum_{j \in I_{s}} K_{j}
$$

$Z_{s}$ имеет смысл числа исходов серии испытаний, в которых дискретная случайная величина $n$ принадлежит интервалу $I_{s}$. Обобщением (39) является следующая вероятность:

$$
\begin{aligned}
\mathscr{P} & \left(x_{1} \in \Omega_{1}, \ldots, x_{N} \in \Omega_{N} ; Z_{1}, Z_{2}, \ldots\right) \\
& =\sum_{\alpha}^{\prime} \int_{\Omega_{1}} \cdots \int_{\Omega_{N}} d x_{1} \cdots d x_{N}\left|\Phi_{\alpha}\left(x_{1}, \ldots, x_{N}\right)\right|^{2}
\end{aligned}
$$

где через $\sum_{\alpha}^{\prime}$ обозначено суммирование по таким наборам $\alpha=$ $\left(K_{0}, K_{1}, K_{2}, \ldots\right)$, для которых выполняется $(40)$. Для того чтобы получить вероятность $\mathscr{P}\left(Z_{1}, Z_{2}, \ldots\right)$ распределения дискретной переменной по интервалам, в формуле (41) надо положить $\Omega_{j}=\mathbf{R}^{3}, j=1, \ldots, N$. Пусть $p_{j}=p_{i}=P_{I_{s}}$, если $i, j \in I_{s}$. В этом случае имеет место следуюцая теорема. 
Теорема 4. Вероятность $\mathscr{P}\left(Z_{1}, Z_{2}, \ldots\right)$ в случае симметричного ансамбля имеет вид

$$
\mathscr{P}\left(Z_{1}, Z_{2}, \ldots\right)=A \prod_{s=1}^{\infty} \frac{\left(P_{I_{s}}\right)^{Z_{s}}\left(Z_{s}+G_{s}-1\right) !}{Z_{s} !\left(G_{s}-1\right) !},
$$

а в антисимметричном случае

$$
\mathscr{P}\left(Z_{1}, Z_{2}, \ldots\right)=B \prod_{s=1}^{\infty} \frac{\left(P_{I_{s}}\right)^{Z_{s}} G_{s} !}{Z_{s} !\left(G_{s}-Z_{s}\right) !}
$$

где $A$ и В определяются из условия нормировки.

Для доказательства теоремы достаточно воспользоваться следующими тождествами:

$$
\sum_{\substack{K_{1}=0 \\ K_{1}+\cdots+K_{G}=Z}}^{\infty} \ldots \sum_{\substack{K_{G}=0 \\ Z}}^{\infty} 1=\frac{(Z+G-1) !}{Z !(G-1) !}, \quad \sum_{\substack{K_{1}=0 \\ K_{1}+\cdots+K_{G}=Z}}^{1} \ldots \sum_{\substack{K_{G}=0 \\ Z !(G-Z) !}}^{1} 1=\frac{G !}{Z !}
$$

Формулы (42), (43) хорошо известны в статистической физике и используются для вывода распределений Бозе-Эйнштейна и Ферми-Дирака [1]. Заметим, что аналогичные вероятности для случая ансамбля без условия симметрии или антисимметрии имеют вид

$$
\mathscr{P}\left(Z_{1}, Z_{2}, \ldots\right)=C \prod_{s=1}^{\infty} \frac{\left(P_{I_{s}} G_{s}\right)^{Z_{s}}}{Z_{s} !} .
$$

Это выражение также хорошо известно и соответствует распределению Больцмана.

Пусть $\Phi_{\alpha}\left(x_{1}, \ldots, x_{N}\right)$ - полная ортогональная система симметричных функций в симметричном подпространстве пространства $L_{2}\left(\mathbf{R}^{3 N}\right)$. Предположим, что оператор ансамбля $\widehat{H}_{N}$, определяющий вероятностную задачу, имеет вид

$$
\begin{aligned}
& \left(\widehat{H}_{N} \Phi\right)\left(x_{1}, \ldots, x_{N}\right)=-A_{N} \frac{1}{\gamma} \sum_{\alpha} \frac{\ln p_{\alpha}}{p_{\alpha}} \Phi_{\alpha}\left(x_{1}, \ldots, x_{N}\right) \\
& \quad \times \int \cdots \int d y_{1} \cdots d y_{N} \Phi_{\alpha}\left(y_{1}, \ldots, y_{N}\right) \Phi\left(y_{1}, \ldots, y_{N}\right),
\end{aligned}
$$

где $A_{N}>0$ так зависит от $N$, что $\lim _{N \rightarrow \infty}\left(N A_{N}\right)=$ const $>0$, a $p_{\alpha}$, в соответствии с данным выше определением, такие, что

$$
p_{\alpha}=\int \cdots \int d x_{1} \cdots d x_{N}\left|\Phi_{\alpha}\left(x_{1}, \ldots, x_{N}\right)\right|^{2}, \quad \sum_{\alpha} p_{\alpha}=1 .
$$

Приведем необходимые далее сведения о бозонном $F_{\mathrm{B}}$ и фермионном $F_{\mathrm{F}}$ пространствах Фока [7]. Эти пространства состоят из последовательностей функций $\left\{\psi_{n}\right\}$, где $n=0,1,2, \ldots, \psi_{n}\left(x_{1}, \ldots, x_{n}\right) \in L_{2}\left(\mathbf{R}^{3 n}\right)-$ 
в бозонном случае симметричная, а в фермионном антисимметричная функция. Вакуумный вектор $\Psi_{0}$ - это следующая последовательность: $\Psi_{n}\left(x_{1}, \ldots, x_{n}\right)=0$ при $n>0, \psi_{0}=1$. Операторы рождения и уничтожения $\widehat{\psi}^{+}(x)$ и $\widehat{\psi}^{-}(y)$ определяются следуюшим образом:

$$
\begin{aligned}
& \left(\widehat{\psi}^{-}(x) \Psi\right)_{n}\left(x_{1}, \ldots, x_{n}\right)=\sqrt{n+1} \Psi_{n+1}\left(x, x_{1}, \ldots, x_{n}\right), \\
& \left(\widehat{\psi}^{+}(x) \Psi\right)_{n}\left(x_{1}, \ldots, x_{n}\right) \\
& \quad=\frac{1}{\sqrt{n}} \sum_{s=1}^{n}( \pm 1)^{s-1} \delta\left(x-x_{s}\right) \Psi_{n-1}\left(x_{1}, \ldots, x_{s-1}, x_{s+1}, \ldots, x_{n}\right),
\end{aligned}
$$

где «土» в бозонном случае равен «+», а в фермионном «-». Из (45) следует, что выполняются соотношения

$$
\begin{gathered}
\widehat{\psi}^{-}(x) \Psi_{0}=0 \\
{\left[\widehat{\psi}^{-}(x), \widehat{\psi}^{+}\left(x^{\prime}\right)\right]_{ \pm}=\delta\left(x-x^{\prime}\right), \quad\left[\widehat{\psi}^{ \pm}(x), \widehat{\psi}^{ \pm}\left(x^{\prime}\right)\right]_{ \pm}=0,}
\end{gathered}
$$

где «土» означает, что в бозонном случае рассматривается коммутатор, а в фермионном - антикоммутатор. Кроме того, любой вектор $\Psi$ пространства $F$ представляется в виде:

$$
\Psi=\sum_{s=0}^{\infty} \frac{1}{\sqrt{s !}} \int \cdots \int d x_{1} \cdots d x_{s} \psi_{s}\left(x_{1}, \ldots, x_{s}\right) \widehat{\psi}^{+}\left(x_{1}\right) \cdots \widehat{\psi}^{+}\left(x_{s}\right) \Psi_{0} .
$$

$N$-частичным подпространством пространства $F_{\mathrm{B}}\left(F_{\mathrm{F}}\right)$ называют подпространство, элементы $\Psi$ которого удовлетворяют условию $\psi_{n}\left(x_{1}, \ldots\right.$, $\left.x_{n}\right)=0$, если $n \neq N$.

Рассмотрим функционал, соответствующий оператору (44):

$$
\mathscr{L}\left(\varphi_{1}, \varphi_{2}\right)=\left(\Phi_{1}, \widehat{H}_{N} \Phi_{2}\right),
$$

где $\varphi_{1}, \varphi_{2} \in L_{2}\left(\mathbf{R}^{3}\right), \Phi_{1}\left(x_{1}, \ldots, x_{N}\right)=\varphi_{1}\left(x_{1}\right) \varphi_{1}\left(x_{2}\right) \cdots \varphi_{1}\left(x_{N}\right) \in L_{2}\left(\mathbf{R}^{3 N}\right)$, $\Phi_{2}\left(x_{1}, \ldots, x_{N}\right)=\varphi_{2}\left(x_{1}\right) \varphi_{2}\left(x_{2}\right) \cdots \varphi_{2}\left(x_{N}\right) \in L_{2}\left(\mathbf{R}^{3 N}\right)$, причем функции $\varphi_{1}(x)$ и $\varphi_{2}(x)$ такие, что $\int d x \varphi_{1}(x) \varphi_{2}(x)=1$. Будем предполагать относительно полуплотностей $\Phi_{\alpha}\left(x_{1}, \ldots, x_{N}\right)$, что

1) равенство

$\left.\frac{\delta^{2 s} \mathscr{L}}{\delta \varphi_{1}\left(x_{1}\right) \cdots \delta \varphi_{1}\left(x_{s}\right) \delta \varphi_{2}\left(y_{1}\right) \cdots \delta \varphi_{2}\left(y_{s}\right)}\right|_{\varphi_{1}=\varphi_{2}=0}=I_{s .}\left(x_{1}, \ldots, x_{s} ; y_{1}, \ldots, y_{s}\right)$,

$s=1,2, \ldots$, определяет ядро некоторого самосопряженного оператора $\widehat{C_{s}}$ в $L_{2}\left(\mathbf{R}^{3 s}\right)$ такого, что при $N \rightarrow \infty$ для любой гладкой функции $S\left(x_{1}, \ldots, x_{s}\right)$ и любых финитных гладких функций $\phi_{m}\left(x_{1}, \ldots, x_{s}\right)$, $m=1,2$, матричный элемент $\left(\psi_{1}, \widehat{C_{s}} \psi_{2}\right)$, где

$$
\psi_{m}\left(x_{1}, \ldots, x_{m}\right)=\phi_{m}\left(x_{1}, \ldots, x_{m}\right) \exp \left(i N^{-1 / 3} S\left(x_{1}, \ldots, x_{m}\right)\right),
$$


имеет предел, равный

$$
\begin{gathered}
\int \cdots \int d x_{1} \cdots d x_{s} \phi_{1}^{*}\left(x_{1}, \ldots, x_{m}\right) \phi_{2}\left(x_{1}, \ldots, x_{m}\right) \\
\times D_{s}\left(x_{1}, \ldots, x_{s} ; \frac{\partial S}{\partial x_{1}}, \ldots, \frac{\partial S}{\partial x_{s}}\right)
\end{gathered}
$$

где функция $D_{s}\left(x_{1}, \ldots, x_{s} ; p_{1}, \ldots, p_{s}\right)$ действительная и от функций $\phi_{1,2}$ и $S$ не зависит;

2 ) вариационная производная достаточно высокого порядка по $\varphi_{1}(x)$ и $\varphi_{2}(x)$ от функционала (48) равна нулю.

В этом случае оператору $\widehat{H}_{N}$ сопоставим вторично квантованные операторы в бозонном и фермионном фоковских пространствах:

$$
\begin{aligned}
& \widehat{H}=\frac{1}{N} \iint d x d y \widehat{\psi}^{+}(x) I_{1}(x, y) \widehat{\psi}^{-}(y) \\
& +\frac{1}{N(N-1)} \iiint \int d x_{1} d y_{1} d x_{2} d y_{2} \widehat{\psi}^{+}\left(x_{1}\right) \widehat{\psi}^{+}\left(x_{2}\right) \\
& \quad \times I_{2}\left(x_{1}, x_{2} ; y_{1}, y_{2}\right) \widehat{\psi}^{-}\left(y_{2}\right) \widehat{\psi}^{-}\left(y_{1}\right) \\
& +\sum_{s=3} \frac{(N-s) !}{N !} \int \cdots \int d x_{1} d y_{1} \cdots d x_{s} d y_{s} \widehat{\psi}^{+}\left(x_{1}\right) \cdots \widehat{\psi}^{+}\left(x_{s}\right) \\
& \quad \times I_{2}\left(x_{1}, \ldots, x_{s} ; y_{1}, \ldots, y_{s}\right) \widehat{\psi}^{-}\left(y_{1}\right) \cdots \widehat{\psi}^{-}\left(y_{s}\right) .
\end{aligned}
$$

Имеет место следующая теорема.

Теорема 5. Если функиии $I_{s}\left(x_{1}, \ldots, x_{s} ; y_{1}, \ldots, y_{s}\right)$ не зависят от $N$ при всех $s=1,2, \ldots$, то для любого вектора $\Psi$ справедливо

$$
(\widehat{H} \Psi)_{N}\left(x_{1}, \ldots, x_{N}\right)=\left(\widehat{H}_{N} \Psi_{N}\right)\left(x_{1}, \ldots, x_{N}\right),
$$

где $\widehat{H}$ - onepamop (49), a $\widehat{H}_{N}$ - onepamop (44).

Доказательство теоремы получается непосредственной подстановкой вектора (47) в (31) с использованием свойств (46). Таким образом, можно сказать, что при выполнении условия теоремы оператор (49) на $N$-частичном подпространстве совпадает с оператором (44).

Введем следующий оператор в $L_{2}\left(\mathbf{R}^{3 N}\right)$ :

$$
\widehat{F}_{\theta N}=\widehat{H}_{N}-\theta \widehat{S}_{N}
$$

где $\widehat{H}_{N}$ - оператор $(34)$, а $\widehat{S}_{N}$ - следующий оператор в пространстве $L_{2}\left(\mathbf{R}^{3 N}\right)$ :

$$
\begin{aligned}
\left(\widehat{S}_{N} \Phi\right)\left(x_{1}, \ldots, x_{N}\right) & \\
=\sum_{n_{1}=0}^{\infty} \cdots & \sum_{n_{N}=0}^{\infty} \ln \left(\frac{N !}{\prod_{j=0}^{\infty} K_{j} !}\right) \frac{1}{\prod_{j^{\prime}=0}^{\infty} p_{j^{\prime}}} \Phi_{n_{1}, \ldots, n_{N}}\left(x_{1}, \ldots, x_{N}\right) \\
& \quad \times \int \cdots \int d y_{1} \cdots d y_{N} \Phi_{n_{1}, \ldots, n_{N}}\left(y_{1}, \ldots, y_{N}\right) \Phi\left(y_{1}, \ldots, y_{N}\right),
\end{aligned}
$$


здесь $\Phi_{n_{1}, \ldots, n_{N}}\left(y_{1}, \ldots, y_{N}\right)$ - функции $(32)$, а $K_{j}-$ целые числа, зависящие от $n_{1}, \ldots, n_{N}$ (в формуле (52) эта зависимость опущена) следующим образом:

$$
K_{j}\left(n_{1}, \ldots, n_{N}\right)=\sum_{s=1}^{N} \delta_{j n_{s}},
$$

где $\delta_{j n}$ - символ Кронекера. Будем считать, что в температурном случае полуплотности $\Phi_{\alpha}\left(x_{1}, \ldots, x_{N}\right)$ являются собственными функциями оператора (44), удовлетворяющими условию (36). Симметричное и антисимметричное подпространства $L_{2}\left(\mathbf{R}^{3 N}\right)$ являются инвариантными подпространствами оператора $\widehat{F}_{\theta N}$, поэтому температурный случай тождественных испытаний рассматривается аналогично, только функции $\Phi_{\alpha}\left(x_{1}, \ldots, x_{N}\right)$ являются симметричными или антисимметричными. При рассмотрении симметричного случая удобно пользоваться формализмом вторичного квантования. В монографии автора и О. Ю. Шведова [8] развит метод нахождения асимптотики собственных значений и собственных функций вторично квантованных операторов вида (49) при $N \rightarrow \infty$. Далее в работе эти методы применяются для нахождения асимптотик собственных значений оператора (51).

Чтобы построить асимптотики собственных значений в случае ансамбля систем, каждая из которых описывается бозонным или фермионным оператором (49), рассмотрим представление ультравторичного квантования. Для квантования ансамбля из ультравторично квантованных систем применяется метод третичного квантования [8].

Состояния ультравторично квантованной системы описываются векторами пространства $\mathscr{F}[4]-[6]$. Это пространство является бозонным пространством Фока [7], в нем определены: $\widehat{b}^{+}(x, s)$ - оператор рождения частиц с номером $s, \widehat{b}^{-}(x, s)$ - оператор уничтожения частиц с номером $s$, а также $\widehat{B}^{+}\left(x, x^{\prime}\right)$ - оператор рождения пары частиц, $\widehat{B}^{-}\left(x, x^{\prime}\right)$ - оператор уничтожения пары частиц, далее считаем, что $x, x^{\prime} \in \mathbf{T}, \mathbf{T}-$ трехмерный тор $L \times L \times L, s$ - дискретная переменная, называемая номером или статистическим спином, $s=0,1, \ldots$. Операторы $\widehat{b}^{ \pm}(x, s)$ и $\widehat{B}^{ \pm}\left(x, x^{\prime}\right)$ удовлетворяют стандартным коммутационным соотношениям [7]. Кроме того, в пространстве $\mathscr{F}$ существует вакуумный вектор $\Phi_{0}$ такой, что $\left(\Phi_{0}, \Phi_{0}\right)=1, \widehat{b}^{-}(x, s) \Phi_{0}=0, \widehat{B}^{-}\left(x, x^{\prime}\right) \Phi_{0}=0$, и любой вектор $\Phi$ пространства $\mathscr{F}$ единственным образом представляется в виде

$$
\begin{aligned}
\Phi=\sum_{k=0}^{\infty} & \sum_{M=0}^{\infty} \frac{1}{k ! M !} \sum_{s_{1}=0}^{\infty} \cdots \sum_{s_{k}=0}^{\infty} \int \cdots \int d x_{1} \cdots d x_{k} d y_{1} \cdots d y_{2 M} \\
& \times \Phi_{k, M}\left(x_{1}, s_{1} ; \ldots ; x_{k}, s_{k} ; y_{1}, y_{2} ; \ldots ; y_{2 M-1}, y_{2 M}\right) \\
& \times \widehat{b}^{+}\left(x_{1}, s_{1}\right) \cdots \widehat{b}^{+}\left(x_{k}, s_{k}\right) \widehat{B}^{+}\left(y_{1}, y_{2}\right) \cdots \widehat{B}^{+}\left(y_{2 M-1}, y_{2 M}\right) \Phi_{0},
\end{aligned}
$$


где $\Phi_{k, M}$ - функции, симметричные относительно перестановок пар переменных $\left(x_{i}, s_{i}\right)$ и $\left(x_{j}, s_{j}\right)$ и относительно перестановок пар переменных $\left(y_{2 l-1}, y_{2 l}\right)$ и $\left(y_{2 m-1}, y_{2 m}\right), l, m=1, \ldots, M$.

Спектр энергий ультравторично квантованной системы определяется следующей задачей на собственные значения:

$$
\overline{\widehat{H}} \Phi=\lambda \overline{\widehat{E}} \Phi, \quad \overline{\widehat{E}} \Phi \neq 0
$$

где $\overline{\widehat{H}}, \widehat{\widehat{E}}$ - ультравторично квантованные операторы в пространстве $\mathscr{F}$ :

$$
\begin{aligned}
& \overline{\widehat{H}}=\mathscr{H}\left[\widehat{B}^{+}(\cdot), \widehat{B}^{-}(\cdot), \widehat{b}^{+}(\cdot), \widehat{b}^{-}(\cdot)\right], \\
& \overline{\widehat{E}}=E\left[\widehat{B}^{+}(\cdot), \widehat{B}^{-}(\cdot), \widehat{b}^{+}(\cdot), \widehat{b}^{-}(\cdot)\right] .
\end{aligned}
$$

Здесь $\mathscr{H}$ и $E$ - функционалы, зависящие от функций $\Phi(x, y)$ и $\varphi(x, s)$ и имеющие вид

$$
\begin{aligned}
& \mathscr{H}\left[\Phi^{*}(\cdot), \Phi(\cdot), \varphi^{*}(\cdot), \varphi(\cdot)\right] \\
& =\exp \left(-\iint d x d y \Phi^{*}(x, y) \Phi(x, y)\right. \\
& \left.\quad-\sum_{s=0}^{\infty} \int d x \varphi^{*}(x, s) \varphi(x, s)\right) \operatorname{Sp}(\widehat{H} \widehat{\rho}), \\
& E\left[\Phi^{*}(\cdot), \Phi(\cdot), \varphi^{*}(\cdot), \varphi(\cdot)\right] \\
& =\exp \left(-\iint d x d y \Phi^{*}(x, y) \Phi(x, y)\right. \\
& \left.\quad-\sum_{s=0}^{\infty} \int d x \varphi^{*}(x, s) \varphi(x, s)\right) \operatorname{Sp}(\widehat{\rho}) .
\end{aligned}
$$

В формулах (56) и (57) $\hat{\rho}$ - вторично квантованный оператор, зависящий от $\Phi(x, y)$ и $\varphi(x, s)$; в бозонном случае этот оператор имеет вид

$$
\begin{aligned}
\widehat{\rho}=\sum_{k=0}^{\infty} \sum_{M=0}^{\infty} \sum_{M^{\prime}=0}^{\infty} & \frac{1}{k ! \sqrt{M ! M^{\prime} !(k+2 M) !\left(k+2 M^{\prime}\right) !}} \\
& \times\left(\sum_{s=0}^{\infty} \iint d x d x^{\prime} \varphi(x, s) \varphi^{*}\left(x^{\prime}, s\right) \widehat{\psi}^{+}(x) \widehat{\psi}^{-}\left(x^{\prime}\right)\right)^{k} \\
& \times\left(\iint d y d y^{\prime} B\left(y, y^{\prime}\right) \widehat{\psi}^{+}(y) \widehat{\psi}^{+}\left(y^{\prime}\right)\right)^{M} \\
& \times\left(\iint d z d z^{\prime} \Phi^{*}\left(z, z^{\prime}\right) \widehat{\psi}^{-}(z) \widehat{\psi}^{-}\left(z^{\prime}\right)\right)^{M} \\
& \times \exp \left(-\int d \xi \widehat{\psi}^{+}(\xi) \widehat{\psi}^{-}(\xi)\right)
\end{aligned}
$$


здесь $\widehat{\psi}^{+}(x)$ и $\widehat{\psi}^{-}(x)$ - рассмотренные выше бозонные операторы рождения и уничтожения (45). Кроме того, в формуле (56) $\hat{H}$ - вторично квантованный гамильтониан вида (49), для простоты далее ограничимся случаем парного взаимодействия. В фермионном случае оператор $\widehat{\rho}$ имеет вид

$$
\begin{gathered}
\widehat{\rho}=\sum_{k=0}^{\infty} \sum_{M=0}^{\infty} \frac{1}{k ! M !(k+2 M) !}\left(\iint d y_{1} d y_{2} B\left(y_{1}, y_{2}\right) \widehat{\psi}^{+}\left(y_{1}\right) \widehat{\psi}^{+}\left(y_{2}\right)\right)^{M} \\
\sum_{s_{1}=0}^{\infty} \cdots \sum_{s_{k}=0}^{\infty} \int \cdots \int d x_{1} d x_{1}^{\prime} \cdots d x_{k} d x_{k}^{\prime} \\
\times b\left(x_{1}, s_{1}\right) b^{*}\left(x_{1}^{\prime}, s_{1}\right) \cdots b\left(x_{k}, s_{k}\right) b^{*}\left(x_{k}^{\prime}, s_{k}\right) \\
\times \widehat{\psi}^{+}\left(x_{1}\right) \cdots \widehat{\psi}^{+}\left(x_{k}\right) \widehat{P}_{0} \widehat{\psi}^{-}\left(x_{k}^{\prime}\right) \cdots \widehat{\psi}^{-}\left(x_{1}^{\prime}\right) \\
\times\left(\iint d y_{1}^{\prime} d y_{2}^{\prime} B\left(y_{1}^{\prime}, y_{2}^{\prime}\right) \widehat{\psi}^{-}\left(y_{2}^{\prime}\right) \widehat{\psi}^{-}\left(y_{1}^{\prime}\right)\right)^{M}
\end{gathered}
$$

где в данном случае $\widehat{\psi}^{+}(x), \widehat{\psi}^{-}(x)$ - фермиевские операторы рождения и уничтожения, $\widehat{P}_{0}$ - проектор на вакуумный вектор фермионного фоковского пространства [7].

Для третичного квантования ансамбля далее будет использоваться представление, в котором векторам $\Phi$ пространства $\mathscr{F}$ соответствуют функционалы $\Phi[A(\cdot), q(\cdot)]$ от функций $Q(x, y), q(x, s)$, а операторы $\widehat{B}^{ \pm}(x, y)$ и $\widehat{b}^{ \pm}(x, s)$ имеют вид

$$
\begin{aligned}
& \widehat{B}^{\mp}(x, y)=\frac{1}{\sqrt{2}}\left(Q(x, y) \pm \frac{\delta}{\delta Q(x, y)}\right), \\
& \widehat{b}^{\mp}(x, s)=\frac{1}{\sqrt{2}}\left(q(x, s) \pm \frac{\delta}{\delta q(x, s)}\right) .
\end{aligned}
$$

Скалярное произведение векторов $\Phi_{1}$ и $\Phi_{2}$ в этом представлении есть континуальный интеграл

$$
\left(\Phi_{1}, \Phi_{2}\right)=\int \cdots \int \prod_{x, y} D Q(x, y) \prod_{s=0}^{\infty} \prod_{z} D q(z, s) \Phi_{1}^{*}[Q(\cdot), q(\cdot)] \Phi_{2}^{*}[Q(\cdot), q(\cdot)] .
$$

Прежде чем перейти к рассмотрению ансамбля, отметим, что в соответствии с постулатом о том, что вероятностная задача определяется оператором, мы должны считать, что полуплотности $\Phi_{k}[Q(\cdot), q(\cdot)]$ для ультравторично квантованной системы являются решениями задачи (53). Причем для этих решений выполняются дополнительные условия, аналогичные (36):

$$
\left(\Phi_{k}, \widehat{E} \Phi_{l}\right)= \begin{cases}0 & \text { при } \quad k \neq l, \\ \frac{e^{-\gamma \lambda_{k}}}{\sum_{m} e^{-\gamma \lambda_{m}}} & \text { при } k=l .\end{cases}
$$


Рассмотрим теперь ансамбль из $N$ одинаковых невзаимодействующих ультравторично квантованных систем. Состояния ансамбля описываются векторами $L$ пространства $\mathscr{L}_{N}=\operatorname{Symm} \underbrace{\mathscr{F} \otimes \cdots \otimes \mathscr{F}}_{n \text { штук }}$.

Элементами этого пространства являются функционалы $L\left[Q_{1}(\cdot)\right.$, $\left.q_{1}(\cdot) ; \ldots ; Q_{N}(\cdot), q_{N}(\cdot)\right]$, зависящие от функций $Q_{i}(x, y), q_{i}(x, s), i=$ $1,2, \ldots, N$, и симметричные относительно перестановок пар функций $Q_{i}(x, y), q_{i}(x, s)$ и $Q_{j}(x, y), q_{j}(x, s), i, j=1, \ldots, N$. Так как системы не взаимодействуют, то будем считать, что вероятностная задача для этого ансамбля определяется следующей задачей на собственные значения:

$$
\begin{aligned}
\sum_{i=1}^{N}\left(\prod _ { j = 1 } ^ { N } E \left[\frac{1}{\sqrt{2}}\left(Q_{j}(\cdot)-\frac{\delta}{\delta Q_{j}(\cdot)}\right), \frac{1}{\sqrt{2}}\left(Q_{j}(\cdot)+\frac{\delta}{\delta Q_{j}(\cdot)}\right),\right.\right. \\
\left.\left.\quad \frac{1}{\sqrt{2}}\left(q_{j}(\cdot)-\frac{\delta}{\delta q_{j}(\cdot)}\right), \frac{1}{\sqrt{2}}\left(q_{j}(\cdot)+\frac{\delta}{\delta q_{j}(\cdot)}\right)\right]\right) \\
\times \mathscr{H}\left[\frac{1}{\sqrt{2}}\left(Q_{i}(\cdot)-\frac{\delta}{\delta Q_{i}(\cdot)}\right), \frac{1}{\sqrt{2}}\left(Q_{i}(\cdot)+\frac{\delta}{\delta Q_{i}(\cdot)}\right)\right. \\
\quad \times L\left[Q_{1}(\cdot), q_{1}(\cdot) ; \ldots ; Q_{N}(\cdot), q_{N}(\cdot)\right] \\
=\Lambda \prod_{i=1}^{N} E\left[\frac{1}{\sqrt{2}}\left(Q_{i}(\cdot)-\frac{\delta}{\delta Q_{i}(\cdot)}\right), \frac{1}{\sqrt{2}}\left(Q_{i}(\cdot)+\frac{\delta}{\delta Q_{i}(\cdot)}\right)\right. \\
\left.\quad \frac{1}{\sqrt{2}}\left(q_{i}(\cdot)-\frac{\delta}{\delta q_{i}(\cdot)}\right), \frac{1}{\sqrt{2}}\left(q_{i}(\cdot)+\frac{\delta}{\delta q_{i}(\cdot)}\right)\right] \\
\times L\left[Q_{1}(\cdot), q_{1}(\cdot) ; \ldots ; Q_{N}(\cdot), q_{N}(\cdot)\right] .
\end{aligned}
$$

Будем теперь считать, что число $N$ не фиксировано. В таком случае уравнение (62) может быть третично проквантовано [8]. Если $N$ - переменная величина, то пространство состояний такого ансамбля есть фоковское пространство $\mathscr{L}=\bigoplus_{k=0}^{\infty} \mathscr{L}_{k}$. В этом пространстве вводятся операторы рождения $\widehat{\mathscr{A}}^{+}[Q(\cdot), q(\cdot)]$ и уничтожения $\widehat{\mathscr{A}}^{-}[Q(\cdot), q(\cdot)]$. Уравнение (62) может быть записано в виде

$$
\begin{aligned}
& \left(\iint \prod_{x, y} D Q(x, y) \prod_{s=0}^{\infty} \prod_{z} D q(z, s) \cdot \stackrel{2}{\mathscr{A}}^{+}[Q(\cdot), q(\cdot)]\right. \\
& \quad \times \mathscr{H}\left[\frac{1}{\sqrt{2}}\left(Q(\cdot)-\frac{\delta}{\delta Q(\cdot)}\right), \frac{1}{\sqrt{2}}\left(Q(\cdot)+\frac{\delta}{\delta Q(\cdot)}\right),\right.
\end{aligned}
$$




$$
\begin{aligned}
&\left.\left.\frac{1}{\sqrt{2}}\left(q(\cdot)-\frac{\delta}{\delta q(\cdot)}\right), \frac{1}{\sqrt{2}}\left(q(\cdot)+\frac{\delta}{\delta q(\cdot)}\right)\right] \frac{1}{\mathscr{A}}-[Q(\cdot), q(\cdot)]-\Lambda\right) \\
& \times \exp \left(\iint \prod_{x^{\prime}, y^{\prime}} D Q^{\prime}\left(x^{\prime}, y^{\prime}\right) \prod_{s^{\prime}=0}^{\infty} \prod_{z^{\prime}} D q^{\prime}\left(z^{\prime}, s^{\prime}\right)\right. \\
& \times\left(\frac{2}{\mathscr{A}}+\left[Q^{\prime}(\cdot), q^{\prime}(\cdot)\right] \cdot E\left[\frac{1}{\sqrt{2}}\left(Q^{\prime}(\cdot)-\frac{\delta}{\delta Q^{\prime}(\cdot)}\right), \frac{1}{\sqrt{2}}\left(Q^{\prime}(\cdot)+\frac{\delta}{\delta Q^{\prime}(\cdot)}\right),\right.\right. \\
&\left.\times \frac{1}{\sqrt{2}}\left(q^{\prime}(\cdot)-\frac{\delta}{\delta q^{\prime}(\cdot)}\right), \frac{1}{\sqrt{2}}\left(q^{\prime}(\cdot)+\frac{\delta}{\delta q^{\prime}(\cdot)}\right)\right] \frac{1}{\mathscr{A}}-\left[Q^{\prime}(\cdot), q^{\prime}(\cdot)\right] \\
&\left.\left.-\frac{2}{\mathscr{A}}+\left[Q^{\prime}(\cdot), q^{\prime}(\cdot)\right] \frac{1}{\mathscr{A}}-\left[Q^{\prime}(\cdot), q^{\prime}(\cdot)\right]\right)\right) L=0,
\end{aligned}
$$

где $L-$ вектор пространства $\mathscr{L}$. Уравнение (53) имеет набор решений

$$
\Phi_{k} \in \mathscr{F}, \quad \lambda_{k}, k=0,1, \ldots
$$

такой, что

1) $\left(\Phi_{k}, \overline{\widehat{E}} \Phi_{l}\right)=p_{k} \delta_{k l},\left(\Phi_{k}, \Phi_{l}\right)=p_{k} \delta_{k l}, p_{k}=e^{-\gamma \lambda_{k}} / \sum_{m} e^{-\gamma \lambda_{m}}$;

2) $\left(\Phi_{k}, \widehat{\widehat{E}} \Phi\right)=0$ при всех $k$ тогда и только тогда, когда $\overline{\widehat{E}} \Phi=0$. Заметим также, что $\widehat{\widehat{E} \widehat{H}}=\overline{\widehat{H} \widehat{E}}=\widehat{\widehat{H}}$. Пусть $\Phi_{k}[Q(\cdot), q(\cdot)]$ - отвечающие векторам функционалы. Введем в пространстве $\mathscr{L}$ операторы

$$
\begin{aligned}
& \widehat{a}_{k}^{-}=\frac{1}{\sqrt{p_{k}}} \iint \prod_{x, y} D Q(x, y) \prod_{s=0}^{\infty} \prod_{z} D q(z, s) \Phi_{k}^{*}[Q(\cdot), q(\cdot)] \widehat{\mathscr{A}}^{-}[Q(\cdot), q(\cdot)] \\
& \widehat{a}_{k}^{+}=\frac{1}{\sqrt{p_{k}}} \iint \prod_{x, y} D Q(x, y) \prod_{s=0}^{\infty} \prod_{z} D q(z, s) \Phi_{k}[Q(\cdot), q(\cdot)] \widehat{\mathscr{A}}^{+}[Q(\cdot), q(\cdot)] .
\end{aligned}
$$

Уравнение (63) тогда может быть записано в виде

$$
\begin{aligned}
& {\left[\left[\sum_{k=0}^{\infty} \lambda_{k} \widehat{a}_{k}^{+} \widehat{a}_{k}^{-}-\Lambda\right]\right]} \\
& \times \exp \left(\sum_{k=0}^{\infty} \widehat{a}_{k}^{+} \stackrel{1}{\widehat{a}}_{k}^{-}-\iint \prod_{x, y} D Q(x, y) \prod_{s=0}^{\infty} \prod_{z} D q(z, s) \frac{3}{\mathscr{A}}+\right. \\
& \left.\times[Q(\cdot), q(\cdot)] \frac{1}{\mathscr{A}}-[Q(\cdot), q(\cdot)]\right) L=0 .
\end{aligned}
$$

Выше в этой работе для ансамбля введен оператор $\widehat{S}_{N}(52)$. Обоб- 
щение на ультравторично квантованный случай дает выражение:

$$
\begin{aligned}
& {\left[\left[\ln \left(\frac{\Gamma(\widehat{N}+1)}{\prod_{k=0}^{\infty} \Gamma\left(\widehat{N}_{k}+1\right)}\right)\right]\right]} \\
& \times \exp \left(\sum_{k=0}^{\infty} \widehat{a}_{k}^{+} \widehat{\widehat{a}}_{k}^{-}-\iint \prod_{x, y} D Q(x, y) \prod_{s=0}^{\infty} \prod_{z} D q(z, s) \stackrel{3}{\mathscr{A}}+\right. \\
& \left.\times[Q(\cdot), q(\cdot)] \widehat{\mathscr{A}}^{\frac{1}{}}-[Q(\cdot), q(\cdot)]\right),
\end{aligned}
$$

где $\Gamma(\cdot)$ - гамма-функция Эйлера, $\widehat{N}_{k}=\widehat{a}_{k}^{+} \widehat{a}_{k}^{-}, \widehat{N}=\sum_{k=0}^{\infty} \widehat{N}_{k}$. Кроме того, обобщая (50), будем считать, что при температуре $\theta$ полуплотности для ансамбля определяются уравнением

$$
\begin{aligned}
& {\left[\left[\sum_{k=0}^{\infty} \lambda_{k}\left(\widehat{N}_{k}+\theta \ln \left(\Gamma\left(\widehat{N}_{k}+1\right)\right)\right)-\theta \ln \left(\Gamma\left(\widehat{N}_{k}+1\right)\right)-F\right]\right]} \\
& \times \exp \left(\sum_{k=0}^{\infty} \widehat{3}_{k}^{+} \hat{a}_{k}^{-}-\iint \prod_{x, y} D Q(x, y) \prod_{s=0}^{\infty} \prod_{z} D q(z, s) \stackrel{3}{\mathscr{A}}^{+}\right. \\
& \left.\times[Q(\cdot), q(\cdot)] \stackrel{1}{\mathscr{\mathscr { A }}^{-}}-[Q(\cdot), q(\cdot)]\right) .
\end{aligned}
$$

Если число систем ансамбля $N$ стремится к бесконечности, то в уравнении (67) эвристически можно считать, что операторы $\widehat{\mathscr{A}}^{+}, \widehat{\mathscr{A}}^{-}$ коммутируют [8]. Тогда собственные значения $F$ в уравнении (67) являются экстремальными значениями следующего функционала, зависящего от функционала $A[Q(\cdot), q(\cdot)]$ :

$$
F\left[A^{*}[\cdot], A[\cdot]\right]=\sum_{k=0}^{\infty}\left(\lambda_{k}+\theta \ln \left(\frac{a_{k}^{*} a_{k}}{N}\right)\right) a_{k}^{*} a_{k}
$$

где

$$
a_{k}=\frac{1}{\sqrt{p_{k}}} \iint \prod_{x, y} D Q(x, y) \prod_{s=0}^{\infty} \prod_{z} D q(z, s) \Phi_{k}^{*}[Q(\cdot), q(\cdot)] A[Q(\cdot), q(\cdot)] .
$$

При этом экстремум функционала (68) рассматривается при дополнительном условии

$$
\sum_{k=0}^{\infty} a_{k}^{*} a_{k}=N
$$

Оказывается, что экстремальные значения функционала (68) при условии (70) совпадают с точностью до множителя $N$ с экстремальными 
значениями следующего функционала на пространстве $\mathscr{F}$ :

$$
F[\Phi]=\frac{(\Phi \overline{\widehat{H}} \Phi)}{(\Phi \overline{\widehat{E}} \Phi)}-\theta S[\Phi] .
$$

В формуле $(71) S[\Phi]$ - следующий функционал на пространстве $\mathscr{F}:$

$$
S[\Phi]=-\operatorname{Sp}(\widehat{R}(\Phi) \ln (\widehat{R}(\Phi))),
$$

а $\widehat{R}(\Phi)$ - вторично квантованный оператор вида

$$
\begin{aligned}
& \widehat{R}(\Phi)=\left\{\sum_{k=0}^{\infty} \sum_{M=0}^{\infty} \sum_{M^{\prime}=0}^{\infty} \frac{1}{k ! \sqrt{M ! M^{\prime} !(k+2 M) !\left(k+2 M^{\prime}\right) !}}\right. \\
& \sum_{s_{1}=0}^{\infty} \cdots \sum_{s_{k}=0}^{\infty} \int \cdots \int d x_{1} d x_{1}^{\prime} \cdots d x_{k} d x_{k}^{\prime} d y_{1} \cdots d y_{2 M} d z_{1} \cdots d z_{2 M^{\prime}} \\
& \times \widehat{\psi}^{2}+\left(y_{1}\right) \cdots \widehat{\psi}^{2}+\left(y_{2 M}\right) \widehat{\psi}^{+}+\left(x_{1}\right) \cdots \widehat{\psi}^{2}+\left(x_{k}\right) \\
& \times \frac{1}{\hat{\psi}^{-}}-\left(x_{k}^{\prime}\right) \cdots \frac{1}{\widehat{\psi}^{-}}-\left(x_{1}^{\prime}\right) \frac{1}{\hat{\psi}}-\left(z_{2 M^{\prime}}\right) \cdots \frac{1}{\widehat{\psi}^{-}}-\left(z_{1}\right) \\
& \times \exp \left(-\int d \xi \widehat{\widehat{\psi}}^{+}+(\xi) \widehat{\psi}^{1}-(\xi)\right) \\
& \times\left(\Phi, \stackrel{4}{b}^{+}\left(x_{1}, s_{1}\right) \cdots \stackrel{4}{\widehat{b}^{+}}+\left(x_{k}, s_{k}\right) \stackrel{4}{\widehat{B}}+\left(y_{1}, y_{2}\right) \cdots \stackrel{4}{\widehat{b}}+\left(y_{2 M-1}, y_{2 M}\right)\right. \\
& \times \exp \left(-\sum_{s=0}^{\infty} \int d x \widehat{\vec{b}}^{+}+(x, s) \widehat{3}^{\frac{3}{b}}-(x, s)\right. \\
& \left.-\iint d y d y^{\prime} \stackrel{4}{\widehat{B}}^{+}\left(y, y^{\prime}\right) \widehat{3}^{-}-\left(y, y^{\prime}\right)\right) \\
& \times \stackrel{4}{\widehat{B}}-\left(z_{2 M^{\prime}-1}, z_{2 M^{\prime}}\right) \cdots \widehat{B}^{4}-\left(z_{2}, z_{1}\right) \\
& \left.\left.\times \widehat{b}^{3}-\left(x_{k}^{\prime}, s_{k}\right) \cdots \widehat{b}^{3}-\left(x_{1}^{\prime}, s_{1}\right) \Phi\right)\right\}(\Phi, \overline{\widehat{E}} \Phi)^{-1} \text {. }
\end{aligned}
$$

Векторы, на которых функционал (71) принимает экстремальные значения, имеют вид

$$
\Phi=\sum_{k=0}^{\infty} c_{k} p_{k}^{1 /(2 \theta)-1 / 2} \Phi_{k}
$$

где числа $c_{k}$ принимают значения 0 или 1 . Соответствующее вектору (74) экстремальное значение функционала (71) есть

$$
F=-\theta \ln \left(\sum_{k=0}^{\infty} c_{k} e^{-\lambda_{k} / \theta}\right)
$$


Положим теперь, что в (71) все ультравторично квантованные операторы рождения и уничтожения $\widehat{B}^{-}(x, y), \widehat{B}^{+}(x, y), \widehat{b}^{-}(x, s), \widehat{b}^{+}(x, s)$ можно считать коммутирующими, и заменим их на числовые функции $\Phi(x, y)$, $\Phi^{*}(x, y), \varphi(x, s), \varphi^{*}(x, s)$ соответственно. При этом получим функционал, зависящий от $\Phi$ и $\varphi$. Будем искать экстремумы этого функционала. Оказывается [9], что уравнения экстремума могут быть приведены к виду, совпадающему с уравнениями Бардина-Купера-Шриффера в теории сверхпроводимости [1]. Такой способ получения асимптотик экстремальных значений функционала (71) является эвристическим. В следуюшей части статьи будет приведено строгое доказательство асимптотики при $K, M \rightarrow \infty$, где $K$ и $M-$ собственные значения операторов

$$
\widehat{K}=\sum_{s=0}^{\infty} \int d x \widehat{b}^{+}(x, s) \widehat{b}^{-}(x, s), \quad \widehat{M}=\iint d y d y^{\prime} \widehat{B}^{+}\left(y, y^{\prime}\right) \widehat{B}^{-}\left(y, y^{\prime}\right)
$$

соответственно. В заключение первой части отметим, что выше рассмотрены задачи с одним типом частиц. Однако все сказанное обобщается на случай, когда имеются частицы разных типов.

Автор выражает благодарность В. Н. Батурину и С. Г. Лебедеву за постановку финансовых задач, которые привели к переосмыслению с позиций квантовой статистики задач теории вероятностей.

\section{СПИСОК ЛИТЕРАТУРЫ}

1. Ландау Л. Д., Лифиич Е. М. Статистическая физика. М.: Наука, 1976.

2. Ландау Л. Д., Лифиич Е. М. Квантовая механика. М.: Наука, 1976.

3. Маслов $B$. П. Ультратретичное квантование термодинамики. - Теорет. и матем. физика, 2002, т. 132, № 3, с. 388-398.

4. Маслов В. П. Обобщение метода вторичного квантования на случай специальных тензорных произведений пространств Фока и квантование свободной энергии. Функц. анализ и его прилож., 2000, т. 34, № 4, с. 35-48.

5. Маслов В. П. О способе осреднения для большого числа кластеров. Фазовые переходы. - Теорет. и матем. физика, 2000, т. 125, № 2, с. 297-314.

6. Маслов В.П. Ультравторичное квантование и «ду́хи» в квантованной энтропии. - Теорет. и матем. физика, 2001, т. 129, № 3, с. 464-490.

7. Березин Ф. А. Метод вторичного квантования. М.: Наука, 1987.

8. Маслов В. П., Шведов О.Ю. Метод комплексного ростка в задаче многих частиц квантовой теории поля. М.: Эдиториал УРСС, 2000, 360 с.

9. Маслов В.П. Квантование термодинамики и ультравторичное квантование. М.: Институт компьютерных исследований, 2001, 384 с. 